\title{
EVALUACIÓN DEL IMPACTO DEL SERVICIO DE ASESORÍA PARA ALUMNOS UNIVERSITARIOS EN RIESGO POR BAJO RENDIMIENTO ACADÉMICO
}

\section{IMPACT ASSESSMENT OF THE UNIVERSITY STUDENT COUNSELING SERVICE FOR STUDENTS RISK DUE TO POOR ACADEMIC PERFORMANCE}

\author{
Paola Muñoz Valera* \\ Universidad Peruana de Ciencias Aplicadas (UPC), Perú
}

\section{RESUMEN}

Se presenta la evaluación del servicio tutorial que se brinda a los alumnos de la Universidad Peruana de Ciencias Aplicadas (UPC) que tienen dificultades académicas y, por ello, se encuentran en riesgo de ser separados de la universidad. El objetivo del estudio es indagar, desde un enfoque cualitativo, la percepción que tienen los estudiantes sobre el servicio de asesoría de riesgo académico, en función de la condición de asistencia y superación de riesgo. Se realizaron entrevistas semiestructuradas a 24 estudiantes en situación de riesgo académico sobre su percepción en base a cuatro dimensiones: dimensión general del servicio, metodología, facilitador y sugerencias. Los resultados reflejan que en general los alumnos tienen una percepción favorable del servicio, sobre todo en lo relacionado al apoyo brindado por la universidad y por el facilitador; además brindan sugerencias para mejorar el servicio. Los resultados son discutidos en base al planteamiento teórico sobre la acción tutorial en la universidad.

Palabras clave: evaluación de impacto, tutoría, rendimiento académico, estudiantes universitarios.

\begin{abstract}
This article presents the evaluation of the UPC Student Tutorial Service of for students with a poor academic performance and who are at risk of being dismissed from the university. This study aims at investigating the students perception of the tutorial service when they are at academic risk, taking into account their attendance and how they overcame the risk. Semi-structured interviews were carried out with 24 students at academic risk and the study of their perceptions was based on four dimensions: general services, methodology, facilitator and suggestions. The results reveal that most students regardless of their risk status or attendance, appreciate the service, especially the support provided by the university and the facilitator as well as providing their suggestions to implement the service. The results are discussed based on the theoretical approach of the tutorial services provided.
\end{abstract}

Keywords: impact assessment, tutoring, academic performance, college students. 


\section{INTRODUCCIÓN}

El inicio de los estudios universitarios trae consigo una serie de retos para los jóvenes a nivel tanto académico como personal (GilAlbarova, Martínez, Tunnicliffe \& Moneo, 2013). Para afrontarlos el estudiante debe utilizar y desarrollar estrategias que le permitan hacer frente a estos desafíos, de tal manera que pueda asegurar su permanencia en la universidad.

Dentro de las situaciones académicas, personales y sociales a las que los estudiantes universitarios deben hacer frente se encuentran: la acomodación de la vida personal a los requerimientos de la vida universitaria, el manejo de las habilidades comunicativas, el establecimiento de grupos y redes de soporte (académico y personal) (Cardona, Ramírez \& Tamayo, 2011), así como el despliegue de herramientas y estrategias académicas que le permitan responder de forma satisfactoria a las exigencias de las materias que cursan (HerreraTorres \& Lorenzo-Quiles, 2009). Con respecto a ello, se ha encontrado que el mayor índice de estrés en universitarios se presenta durante los primeros años de la carrera (Muñoz, 1999).

Lamentablemente, muchas de las habilidades necesarias para hacer frente a estos factores no han sido desarrolladas en el colegio (Gil-Albarova, Martínez, Tunnicliffe \& Moneo, 2013). Si los estudiantes no logran encontrar el espacio y las herramientas para desarrollar estas habilidades, es muy probable que presenten serias dificultades en su rendimiento académico.

Si bien es cierto que se han encontrado diversos factores asociados al rendimiento académico en los alumnos universitarios, como aquellos relacionados con la institución educativa, la motivación intrínseca, la forma de enseñanza de los contenidos, el manejo de métodos de estudio, los problemas económicos, el trabajo, las habilidades básicas de los estudiantes para organizar su tiempo, la capacidad de anticipación y planeación del futuro, el autoconcepto personal y académico, las habilidades sociales, entre otros (Mercado \& Niño, 2012; Artunduaga, 2008; Montero, Villalobos \& Valverde, 2007; Tejedor \& GarcíaValcárcel, 2005); lo importante a tomar en consideración es que el bajo rendimiento académico pone en riesgo la permanencia de los alumnos en la universidad.

La deserción universitaria es un fenómeno común para la gran mayoría de países del mundo (Tejedor \& García-Valcárcel, 2005). Las consecuencias que acarrea no solo afectan al estudiante de manera personal (frustración generada por no haber podido alcanzar la meta que se trazó), sino que también impactan sobre la pérdida de la inversión de los recursos humanos y económicos de la familia, de la comunidad y del país (Mori, 2012).

Himmel (2002) distingue dos tipos de abandono de los estudios en universitarios: el voluntario, que se refiere al abandono de la carrera para iniciar estudios en otra institución o en otra carrera de la misma institución, por motivos laborales, una detención momentánea en los estudios con la intención de retomarlos más adelante, entre otros; y el involuntario, que se encuentra asociado, sobretodo, al fracaso académico. La autora afirma que las cifras del último tipo de abandono ascienden al 15 y $25 \%$ en las universidades norteamericanas y se incrementan en algunas carreras e instituciones en universidades de Latinoamérica, como por ejemplo en Chile.

En la universidad donde se ha realizado el estudio, esta realidad no es ajena. Cada semestre académico aproximadamente el $13 \%$ del total de estudiantes se encuentra en situación de riesgo académico. Estar en esta situación significa que el alumno podría ser retirado de la universidad 
el semestre consecutivo por presentar de forma reiterada bajo rendimiento académico.

Según el Reglamento de Estudios (Universidad Peruana de Ciencias Aplicadas, 2013a) un alumno se encuentra en riesgo académico cuando: a) obtiene dos promedios ponderados desaprobados en forma consecutiva; b) obtiene tres promedios ponderados desaprobados en forma alterna; c) desaprueba una asignatura por segunda vez; d) obtiene carta de permanencia excepcional; e) se matricula en el límite de ciclos establecido.

El alumno que no supera la condición de riesgo académico en la que se encuentra al finalizar el semestre de estudios que está cursando, es retirado de la universidad.

Ante este panorama y siguiendo el precepto de que toda institución de educación superior tiene como función promover la educación de los alumnos de forma integral (Fernández \& Escribano, 2008) a través de la prestación de un servicio educativo de calidad (Artunduaga, 2008) y dando respuesta a las necesidad de buscar la implementación de acciones que estén orientadas a mejorar el rendimiento académico de los estudiantes (Montero, Villalobos \& Valverde, 2007); se creó en el año 1998 un servicio de asesoría de riesgo académico (Universidad Peruana de Ciencias Aplicadas, 2013b).

La creación de este servicio tuvo como finalidad brindar orientación a los alumnos en riesgo académico para que puedan superar esta condición y continúen con sus estudios universitarios.

Este servicio es coordinado por el área de Orientación de la Dirección de Calidad Educativa (cuyas funciones están orientadas a velar y promover el bienestar del alumno y su adaptación satisfactoria al sistema universitario) y es ejecutado por asesores de riesgo académico (docentes de las distintas carreras) quienes trabajan con los alumnos en riesgo con miras a fomentar el análisis y la toma de conciencia de su situación, guiando la exploración de alternativas de acción y monitoreándolos de cerca para asegurarse de que mejoren su rendimiento y superen la condición académica en la que se encuentran (Universidad Peruana de Ciencias Aplicadas, 2013b).

Los lineamientos generales que rigen el programa están basados en los conceptos básicos del trabajo tutorial universitario (Ariza \& Ocampo, 2005; Fernández \& Escribano, 2008; Malbrán, 2004); es decir se contempla la orientación de tipo académica, personal-afectiva, profesional-vocacional y administrativa (trámites académicos); ya que es necesario desarrollar programas tutoriales que den respuesta a las dificultades que los estudiantes enfrentan en las diferentes dimensiones de su vida (Ariza \& Ocampo, 2005). Además, es de carácter voluntario y busca promover la autonomía en los alumnos (Arbizu, Lobato \& Del Castillo, 2005).

Como parte del servicio, se ejecutan distintas actividades que colaboran a la consecución de los objetivos previamente planteados, entre ellas se encuentran: el Taller de Asesoría de Matrícula, las atenciones (asesorías) presenciales y virtuales, entrevistas con directores, profesores y padres de familia (en caso sea necesario) y elaboración de informes de atención de alumnos.

Previamente al período de matrícula los asesores llevan a cabo talleres de asesoría de matrícula (uno por carrera), su duración es de dos horas y su objetivo es informar y orientar al alumno sobre el servicio de asesoría de riesgo académico y la realización de un proceso de matrícula responsable. 
Durante el semestre los alumnos en riesgo académico tienen la oportunidad de ser atendidos individualmente, de forma presencial o no presencial (virtual) por sus asesores de carrera. Las atenciones presenciales se realizan en los horarios asignados a los asesores en el espacio determinado para este fin, a donde los alumnos pueden acudir en el momento que lo deseen dentro del horario de atención de su asesor. Asimismo, los alumnos en riesgo también pueden realizar consultas a través del correo electrónico institucional, el cual es revisado continuamente por los asesores para poder dar respuesta a sus inquietudes.

Se buscan espacios individuales en la medida en que se ha encontrado que estos permiten conocer la situación del alumno, facilitan que asuman sus responsabilidades, promueven la orientación en la planificación y permiten que demuestren sus emociones (Argüis et al., 2001, como se citó en Ariza \& Ocampo, 2005).

Durante el semestre académico, los asesores de riesgo también atienden las consultas de los alumnos con respecto a una serie de trámites administrativos que pueden realizar con la finalidad de evitar la baja académica; entre estos se encuentran los retiros de cursos y de ciclo, por ejemplo.

Cada semestre, las atenciones llegan a cubrir al $30 \%$ de los alumnos en situación de riesgo académico. Ya que la asistencia al servicio es voluntaria, los estudiantes acuden al número de sesiones que creen convenientes, estas suelen fluctuar entre una y cuatro.

En líneas generales, a través de las actividades y procedimientos descritos previamente, el servicio presentado busca brindar orientación y seguimiento a los alumnos con la condición específica de un bajo rendimiento académico, tratando de cubrir sus necesidades y promover una experiencia de vida universitaria saludable, que permita expresar al máximo las potencialidades del aprendizaje (Hojas, Anais, Bustos, Letelier \& Zuzulich, 2012).

Sin embargo, no se puede dejar de considerar que un programa de orientación para alumnos debe tener un adecuado funcionamiento y eficacia, por lo que es necesario realizar un seguimiento y evaluación continua del mismo (Ariza \& Ocampo, 2005). Es por ello que durante el primer semestre del presente año se realizó un estudio de enfoque cualitativo con el objetivo de indagar acerca de la percepción que tienen los estudiantes sobre el servicio de asesoría de riesgo académico, en función de la condición de asistencia y superación de este problema.

Para ello se realizaron entrevistas telefónicas semiestructuradas a estudiantes en riesgo con el fin de recoger su percepción y sugerencias con respecto a diversos factores del servicio.

\section{Método}

\section{Tipo y diseño de estudio}

El estudio se realizó desde la perspectiva de la metodología cualitativa, ya que permite conocer de forma individual y profunda la percepción de los estudiantes. Con respecto al diseño, es fenomenológico (Creswell, Hanson, Clark \& Morales, 2007).

\section{Participantes}

Los participantes fueron 24 alumnos que han tenido la condición de riesgo académico, a los cuales se ha organizado en cuatro grupos muestrales en función de la condición de asistencia y superación de riesgo académico, tales como se describe a continuación: 
Tabla 1

Distribución muestral según condición de asistencia y superación de riesgo académico

\begin{tabular}{ccc}
\hline Asistencia & Superación & Participantes \\
\hline $\begin{array}{c}\text { Asiste al servicio de asesoría } \\
\text { de riesgo académico }\end{array}$ & Supera la condición de riesgo & 8 \\
$\begin{array}{c}\text { No asiste al servicio de } \\
\text { asesoría de riesgo académico }\end{array}$ & Supera la condición de riesgo & 3 \\
& No supera la condición de riesgo & 7 \\
\hline
\end{tabular}

\section{Técnicas de recolección de datos}

La técnica que se empleó fue la entrevista semiestructurada, que consiste en la conversación entre dos personas: un entrevistador y un entrevistado. Esta actividad fue dirigida y registrada por el entrevistador con el objetivo de obtener información (opiniones y sentimientos) que responden al objetivo de estudio. Cada entrevista tuvo una duración promedio de 30 minutos.

La entrevista se orientó a recoger información acerca de la asistencia al servicio de asesoría de riesgo académico, que presentó dos valores: a) asistencia y b) no asistencia. Al interior de cada valor se trabajaron dos subvalores: a) supera la condición de riesgo académico y b) no supera la condición de riesgo académico.

\section{Procedimiento}

Las entrevistas se realizaron vía telefónica, y fueron conducidas por dos psicólogos con experienciaenlaaplicacióndeestetipodetécnicas. Asimismo, se respetaron los principios éticos de confidencialidad, anonimato y máxima reserva de los datos, y se les explicó a los participantes que la entrevista sería grabada y que los datos obtenidos solo serían utilizados para el presente estudio con fines estrictamente académicos.

Al tener la información recabada, se realizó el análisis de contenido a partir de las siguientes dimensiones: a) dimensión general del servicio, b) dimensión de metodología, c) dimensión del facilitador y d) dimensión de sugerencias.

Debe señalarse que la dimensión general del servicio y la dimensión de sugerencias se trabajaron en todos los valores y subvalores, mientras que la dimensión de metodología y dimensión del facilitador solo se investigaron en aquellos estudiantes que asistieron al servicio de asesoría de riesgo académico.

Asimismo, es importante indicar que en el caso de los estudiantes que no asisten (superan o no superan) se investigaron las estrategias empleadas, y en el caso de aquellos que no superan la condición de riesgo académico fue necesario indagar adicionalmente por los motivos que explican la no superación del riesgo.

\section{RESULTADOS}

Los resultados se organizan en función de la variable de estudio y los valores asumidos, por ello, en primer lugar se presentan los hallazgos obtenidos en los estudiantes que asisten al servicio de asesoría de riesgo académico, dividiéndolos de acuerdo a los subvalores trabajados entre aquellos que superan y aquellos que no superan dicha condición. Y en segundo lugar, se presentan los resultados obtenidos en los estudiantes que no asisten, 
dividiéndolos de modo similar entre los que superan o no la situación de riesgo académico. Se considera, para cada uno de estos casos, las dimensiones mencionadas en el método.

Estudiantes que asisten al servicio de asesoría de riesgo académico y superan dicha condición

Con respecto a la dimensión general del servicio, los entrevistados mencionan que una de las principales razones por las que consideran que se encuentran en riesgo académico es la falta de organización, ya sea entre el estudio y el trabajo, o al hacer los trabajos prácticos que dejan los docentes en los cursos.

«... estoy en riesgo por falta de organización, tiempo que es difícil hacer los trabajos que tengo que presentar». «Porque el ciclo pasado se me complicó bastante con las prácticas y no tenía tiempo para nada y tuve que faltar a varios exámenes por motivos de fuerza mayor, y tuve problemas de tiempo porque llegaba a mi casa muy tarde del trabajo».

Solo un estudiante atribuyó como principal causa la «mala suerte», es decir presenta atribuciones de locus de control externo y no logra identificar su propia responsabilidad por estar en condición de riesgo académico: ...«... estoy por mala suerte».

Por otro lado, los estudiantes de este grupo perciben que la universidad los apoya en la superación de su condición de riesgo académico a través de la orientación de los asesores, señalándoles cuáles son los pasos que deben seguir, así como recibiendo charlas para enfrentar el riesgo y algunos talleres.

«Sí, porque hablando con mi asesora ella me recomienda qué cosas hacer y cuáles son los pasos a seguir y trámites que tengo que hacer».
«Sí, creo que sí, con las charlas de riesgo y talleres».

En relación a las motivaciones que tuvieron los estudiantes para asistir al servicio de asesoría de riesgo académico, se encontró que la mayoría concuerda en señalar que asistieron para recibir apoyo y poder salir de la condición de riesgo en la que se encontraban.

«Porque estoy en riesgo, porque el asesor nos va a apoyar».

«... la forma de poder ayudarme con asesores...».

Con respecto a la percepción de los estudiantes sobre si sienten que las asesorías de riesgo académico les han ayudado a superar la dificultad que presentaban, todos respondieron unánimemente que las asesorías les han ayudado de diversas formas, ya sea en brindarles perspectivas diferentes que les permiten analizar mejor su situación y tomar decisiones más efectivas, así como ayudarlos en organizarse y darles información; lo cual es un indicador del grado de satisfacción que tienen los estudiantes con el servicio.

«Sí, yo creo que sí... me han ayudado a tomar bien las decisiones, cuando me retiré y me convenía retirarme o el problema no era tan grande y podía seguir».

«Sí, en parte son útiles, porque al hablar... hace que me dé una perspectiva diferente». «Sí, bastante en organizarme mejor con el tiempo en algunos cursos».

En la dimensión metodológica, en cuanto a los temas que se abordaron en las asesorías, los estudiantes señalaron que trataron temas como la organización del tiempo, métodos de estudio, cómo rendir exámenes, cuál es el desempeño académico en general durante el ciclo, orientaciones sobre procedimientos que se deben seguir de acuerdo al reglamento de la universidad. 
«El manejo del tiempo y métodos de estudio...».

«... cómo manejar el tiempo, cómo dar exámenes, cómo vas en el ciclo».

«Sobre todos los procedimientos que indica el reglamento de estudios, los que debemos seguir los alumnos como retiros de ciclo».

En relación a la utilidad de las estrategias trabajadas, la mayoría de los estudiantes concuerda en afirmar su utilidad, sobretodo de aquellas relacionadas a cómo organizar mejor su tiempo, cómo conducirse en el curso para mejorar el rendimiento académico, cómo compatibilizar más las diversas actividades que realizan entre el trabajo y los estudios universitarios.

«Sí y creo que son de gran utilidad como la distribución del tiempo, las horas que brindo a estudiar».

«La de estrategias de estudios y me han ayudado a enfocarme».

Uno de los estudiantes no se posiciona sobre el grado de utilidad de las estrategias, porque considera que la decisión de seguir o no dichas estrategias es de cada estudiante.

«... las estrategias fueron y no fueron de utilidad porque la profesora te da el alcance y al final la decisión es de uno y a veces nosotros no le damos la importancia requerida y nos va mal».

Solo uno de los estudiantes señaló que no recibió ningún tipo de estrategias para salir de la condición de riesgo académico.

«No me brindaron estrategias».

Asimismo, los estudiantes identifican aspectos positivos de las asesorías recibidas: el trato personalizado, el interés en las vivencias que tienen y situaciones que les toca enfrentar, el hecho de sentirse comprendidos y apoyados, y no sentir que están solos frente a lo que significa la condición de estar en riesgo académico.

«Porque es individual, al contacto directo con el alumno, y hay mayor facilidad de entendimiento».

«Como le digo es un apoyo de la universidad y te dicen no estás solo, hay gente que te puede apoyar, le cuentas los problemas y nos ayudan, porque muchos de nosotros trabajamos y estudiamos y no todo el mundo te entiende y en ese aspecto te entiende la universidad».

Al preguntar a los estudiantes sobre los aspectos negativos de la asesoría, todos coinciden en señalar que no encuentran dichos aspectos en el servicio.

Se indagó sobre la metodología empleada por el asesor y si esta cubrió las expectativas de los estudiantes, todos respondieron afirmativamente, es decir sintieron que había un trato cercano y personalizado, un buen nivel de comunicación y que se evidenció un interés para que ellos pudieran superar la condición de riesgo académico en la que se encontraban.

«Sí,... me preguntaba bastante de cómo me estaba yendo...».

«Sí, porque está dispuesto a escucharnos y a ayudarnos».

«Sí, porque pudo sentarse conmigo y conversar y buscar formas para yo mejorar».

También se indagó sobre si los estudiantes creían que las asesorías fueron las que los ayudaron a superar el riesgo académico, las respuestas que brindaron son diversas ya que algunos consideran que efectivamente las asesorías les posibilitaron superar la condición de riesgo, pues les enseñaban a manejar sus horas de estudio. 
«Sí, como le digo, porque me brinda cómo manejar mis horas de estudio».

Sin embargo, otros estudiantes consideran que, si bien es cierto las asesorías les brindan apoyo para superar la condición de riesgo, depende de cada estudiante superar dicha condición o no.

"Creo que las asesorías son como cierta ayuda, el hecho que vayas no te garantiza que vas a salir de riesgo, depende de cada uno».

Respecto a qué otros factores consideran los estudiantes que podrían influenciar para que puedan superar la condición de riesgo, refieren la posibilidad de contar con un mayor apoyo psicológico de especialistas ya que la actitud frente al curso que se lleva puede facilitar o predisponer a desaprobarlo. El repaso del contenido mismo del curso y un mayor asesoramiento y seguimiento de parte de los asesores, así como un mayor compromiso de parte de los estudiantes forman parte de lo necesario para mejorar su rendimiento académico. «Pienso que es un $90 \%$, depende de cada uno, y el otro puede ser el profesor que no te jala, uno mismo es quien se jala, y tal vez para ese curso que yo estoy, es un curso que se dicta de otra carrera y estaba dentro de mi currícula obligatorio y a muchos no nos gusta, y otros cursos sí me gustan, y quizá el contenido del curso puede ser un aspecto que define que puedes llegar al riesgo». «Considero tal vez una planeación de estudio, uncomienzodecicloconuncorrecto seguimiento y compromiso que podrían llevarme a mí a un mayor involucramiento, más que todo constante asesoramiento y seguimiento».

Finalmente, en relación a la dimensión de facilitador, todos los alumnos afirman estar satisfechos con la ayuda brindada por los asesores de riesgo, señalan que les ayudó a asumir las cosas con mayor seriedad y tomar decisiones acertadas.

«Sí... me ayudó a tomar bien la decisión de retirarme o no...».

«Sí... me ayudaron con los horarios, darme y hacer seguimiento y ayudarme a tomar en serio todo...».

Asimismo, señalan que también se sintieron apoyados académicamente por el asesor. Solo un estudiante señaló que no se sintió apoyado, básicamente porque su asesor no conocía sus necesidades y de ello probablemente se puede inferir que no las atendió como el alumno esperaba.

«No, del actual asesor no, porque no tenía conocimiento de mis necesidades».

Las respuestas brindadas por los estudiantes sobre el perfil que consideran que debe tener el asesor de riesgo fueron diversas, algunos señalan la importancia que cuente con una adecuada estructura de personalidad, que sea gentil, comprensivo y paciente, asimismo abierto a la diversidad y que no sea prejuicioso para saber acoger a los estudiantes que provienen de diferentes realidades.

"¿En personalidad? Mi asesora que tuve la veo bastante aceptable, era gentil, comprensiva, paciente».

«Persona que sepa llegar al alumno, de confianza, sea amigable, sincera, que sepa entender las realidades diferentes de los alumnos, porque la población ha crecido bastante y el asesor debe saber llegar a todos y no ser una persona prejuiciosa y ser de carácter».

Otrosseñalan que es muyimportanteque el asesor de riesgo cuente con conocimientos psicológicos, ya que los estudiantes pueden 
presentar diversas problemáticas y contar con dichos conocimientos podría optimizar mucho la asesoría.

«En verdad, si se pone así, debería ser en parte un asesor que tenga conocimientos de la parte psicológica, porque muchos de los factores para llegar al programa es por la mala distribución del tiempo y sería una buena herramienta para que usen los profesores».

Otros estudiantes consideran que lo más importante es que el asesor conozca acerca de los cursos de la carrera y los procesos administrativos de la universidad, para que pueda hacer una asesoría eficiente.

"Que el asesor conozca de los cursos pero también de los procesos administrativos de la universidad».

«Debería saber sobre la realidad de las carreras, de los cursos, a veces hay cursos bien difíciles que no puedes llevar más de dos de estos en el ciclo y te recomiendan cuáles llevar».

Asimismo, señalaron que sea una persona que se encuentre disponible para ayudar a los estudiantes, tanto a nivel presencial como virtual, de tal modo que se cuente con su apoyo permanente.

«Una persona que pueda disponer para poder ayudarme tanto virtual como físicamente o coordinar o tal vez, si así se puede definir, entablar una ayuda constante».

En cuanto a la última dimensión, que corresponde a las sugerencias brindadas por los estudiantes para mejorar el servicio de asesoría de riesgo académico, la mayoría de ellas se centró en una mayor disponibilidad de los asesores. Algunos opinaban que podría haber un asesor permanente, otros que un mismo asesor debería atender dos veces por semana para poderse reunir más veces, otros consideran que se necesita un asesor disponible en la mañana y otro en la tarde, así como es preferible que la asesoría sea presencial y no virtual.

«Debería haber un asesor permanente para todos los alumnos».

«... por ejemplo, en ciclos anteriores me parece que mi asesora solo tiene un día a la semana. Está seis horas seguidas, pero puede estar dos veces a la semana para poder encontrarnos más seguido».

«El tema de horarios puede ser, o un asesor quecubra un horario en la mañanay otro en la tarde, y así tener un diálogo personal no virtual porque con una entrevista es mucho mejor y aparte de ello creo que es lo único que puede ser; y yo sé que ponen bastante de su parte y al final depende de nosotros».

Sin embargo, un grupo menor de alumnos señaló su preferencia por las asesorías virtuales para aquellos que por diversas razones no puedan recibir la asesoría a nivel presencial.

"Que la comunicación pudiera ser por mail, para los que no tenemos mucho tiempo, las asesorías con el profesor pudieran ser por mail, asesoría virtual».

\section{Estudiantes que asisten al servicio de asesoría de riesgo académico y no superan dicha condición}

En la dimensión general, en relación a las razones por las que se encuentran en riesgo académico, la mayoría de los estudiantes señala que fue por falta de organización en los cursos en los cuales se matriculó, así como a interferencias por el horario de trabajo.

«Bueno sí, fue por falta de organización, lo que pasa es que me cambié de carrera, estaba en Ingeniería y me cambié para Economía y Finanzas y creí que 
matemática no me iba a convalidary no me pude retirar y simplemente dejé de asistir y fue por eso que bajó mi ponderado».

«Fue por mala coordinación con mis horarios, el trabajo y la universidad».

Otra de las razones expuestas fue por falta de comprensión de los contenidos que se impartían en el curso.

«El primer tema era porque la evaluación era grupal, era en dos y estuve bien hasta el final y no comprendí bien el tema y fue lo que me bajó demasiado. Al final fue lo de la PC 4 y al final fue lo que me malogró para la segunda y para la primera vez fue porque no entendía nada del curso».

Y otra de las razones fue por la falta de tiempo, ya que consideran que cuatro meses es un tiempo muy corto, asociado a los tipos de temas y la distribución de las horas teóricas y prácticas.

«El primer factor fue el hecho de ver temas muy densos, el primer ciclo me dificultaron el hecho de tener poco tiempo, este fue el primer factor que llevó a complicarme, no a la dificultad sino a la carga teórica, el tiempo muy corto, cuatro meses, y eso sumado a la hora práctica, muy poco tiempo asimilar la teoría y ponerlo en práctica con los ejercicios y trato de buscar apoyo con alguna profesora fuera de la universidad, pero son muy pocos profesores de economía que pueden ayudarte, casi nulo, no hay».

Es decir, las principales razones expuestas por los estudiantes se refieren a la falta de organización, la falta de comprensión del curso, a la brevedad del mismo y a la distribución de las horas de teoría y práctica.

Al preguntar a los estudiantes si sienten que la Universidad de Ciencias Aplicadas les ha brindado las facilidades necesarias para poder superar la condición de riesgo, las respuestas están divididas, por un lado hay estudiantes que responden de modo afirmativo, a pesar que no han logrado superar la condición de riesgo.

«Creo que sí, porque al darme un asesor personal creo que es una posibilidad para superarme».

Pero al mismo tiempo existen estudiantes que consideran que la universidad no les ha brindado todas las facilidades necesarias, específicamente debido al nivel de exigencia académica y las horas de clases que reciben, manifiestan que sería necesario contar con horas de asesoría del profesor fuera de clase sobre los contenidos del curso que necesitan reforzar.

Por otro lado, entre las principales motivaciones que los estudiantes refieren para asistir al servicio de asesoría de riesgo académico, se encuentra el temor de ser retirado de la universidad y que la inversión económica hecha hasta el momento se pierda.

«Lo que me impulsó fue el temor que me sacaran de la universidad, el hecho de que haya avanzado y de un momento a otro me boten por algo que me genera dificultad y todo lo que he invertido se va al agua, y eso fue lo que me impulsó en ir, básicamente eso fue».

Otra de las motivaciones fue el rol de una docente que motivó en forma directa a que asista el estudiante, expresándole comprensión por la situación académica que estaba pasando y brindándole la confianza de que lo podría superar, como se refleja a continuación:

«Porque la profesora que es mi asesora me enseñó anteriormente y sabe cómo yo me manejo en los cursos y cuando le comenté que era por segunda vez, ella me motivó porque me dijo que el tema no era que yo era mala alumna, sino que fácil, 
el curso se me hacía un poco complicado

y comúnmente los alumnos tienen problemas en ese curso...».

En relación a la percepción que tienen sobre el servicio de asesoría de riesgo académico, algunos estudiantes señalan que este les aclaró el panorama académico, el significado de estar en riesgo académico, las implicancias y la decisión que debían tomar al respecto.

«Sí, porque tengo varias dudas y fui $y$ me ayudó a aclarar varias dudas sobre dadas de baja y riesgo académico, el mismo riesgo académico».

Al mismo tiempo, otros estudiantes consideran que en realidad el servicio no les ayuda a superar la dificultad que presentan, sino que les informa sobre lo que significa la condición de estar en riesgo académico para que cada uno tome la decisión que considera pertinente.

"Realmente no, yo lo entiendo de mi perspectiva, como que lo veo que vas y el profesor te da un panorama de la situación que estás pasando [...] y lo demás es de tu parte y está bien, creo que en parte es así, pero en ese momento te sientes presionado por las clases, por las cosas que estás pasando, por los factores académicos [...] y bueno, al final tú tomas la decisión o te retiras de tal curso o te cambias de universidad [...], entonces es como que estás pasando por esto, vas a las asesorías y tú tomas la decisión».

En la dimensión metodológica, en relación a los temas tratados en la asesoría, los estudiantes refieren que los temas más abordados han sido identificar el desempeño académico en los cursos, antes y después de los exámenes parciales.
"Cómo van mis cursos actuales y de acuerdo a mis notas, cómo iba antes del parcial, creo que con lo que me han dado es suficiente porque hablan sobre tu riesgo».

Así también, refieren que es importante que no solo se enfatice en las notas obtenidas, sino sobre todo en el grado de asimilación de los contenidos de un curso y qué tipo de dificultad tienen al respecto.

«Abordamos los cursos que disminuían mi ponderado, me hubiera gustado que aborden los aspectos, por ejemplo, más allá de una nota, sino los aspectos de contenido, digamos por qué no asimilas ese tema, si tienes alguna dificultad, o entiendes o por qué no entiendes al profesor, lo que falta más allá de simplemente ponerlo en cifras».

En lo que respecta a la utilidad de las estrategias empleadas en las asesorías, los estudiantes refieren que en algunos casos la estrategia consistió en estimularlos para que mantengan buenas notas, lo cual evidentemente resultó ser útil.

«...me dieron el consejo de mantener las notas y tratar de llegar a un ponderado alto, y la estrategia que me brindaron fue de utilidad porque hasta el momento sigo con buenas notas».

Por otro lado, otros estudiantes señalan que en realidad no consideran que hayan recibido ninguna estrategia específica.

«Alguna estrategia específica, ninguna, me dijeron simplemente los cursos que más pesan y que debo sacar más nota y bueno eso fue lo que entendí y me dijeron básicamente eso [...]».

Al preguntarle a los estudiantes cuáles creían que habían sido los aspectos positivos de las asesorías recibidas, señalan que el hecho 
de indicarles con claridad que deben ponerle más empeño y dedicación a sus estudios para obtener un mejor rendimiento y así poder salir de la condición de riesgo académico.

«Sí, porque simplemente para salir del riesgo tienes que poner más empeño y sales del riesgo. El aconsejarme y recomendarme que los cursos no generen tanta carga».

Otro aspecto positivo es que se les oriente sobre cuáles son las razones por las que han llegado a esa situación de riesgo y se les puntualice en la nota que deben obtener para salir de dicha situación.

«El hecho de que alguien te diga en qué estas fallando o por qué has estado fallando o que te digan saca tal nota para que puedas salir del riesgo, al menos una orientación».

Al indagar sobre su percepción sobre los aspectos negativos de la asesoría, varios de los estudiantes coincidieron en señalar que no encontraban ninguno.

«Creo que ninguno, en mi caso mi asesora es súper seria, formal, dice las cosas claras y te sabe explicar».

«En la asesoría no tengo ningún aspecto negativo porque en realidad la profesora te apoya...».

Otro de los estudiantes considera que hay un descuido del aspecto académico, no se indaga si es que se asimilan los temas, el estilo del docente, si es comprensible su modo de explicar o si existe disponibilidad de parte del docente para brindar mayores explicaciones.

«Lo negativo, como te dije anteriormente, [...] descuidar el aspecto académico, si se está asimilando correctamente los temas, qué tal enseña el profesor, si el profesor va a clases o no, si lo entiendes o si el profesor de prácticas es un poco reacio a la hora de explicar, y todas esas cosas determinan y tienen un impacto en la nota».

En relación al grado de efectividad del medio a través del cual se realizó la asesoría presencial o virtual, los estudiantes afirman que la asesoría presencial fue efectiva, ya que fue personalizada, directa y respondió a las necesidades que tenía.

«Fue presencial y me parece bien, la asesoría es personalizada, sin mucho palabreo, concisa y era justo lo que quería saber».

En referencia a si la metodología empleada por el asesor cubrió sus expectativas, las opiniones están divididas. Por un lado los estudiantes consideran que sí, que no solo las cubrió sino que incluso las superó hasta el grado de considerarlo como un amigo.

«Sí, más de lo que esperé porque pensé que una asesora no te iba a decir cosas, pero me dijo las cosas claras y como si me estuviera hablando un amigo».

En relación a otros factores que influyeron para que no pueda superar la condición de riesgo académico, los estudiantes señalan las interferencias producidas por el horario de trabajo; y por otro lado, los problemas de salud.

«Más que todo porque yo trabajaba, eso a mí se me desacomodó mucho, por mi horario».

«Aparte de los académicos, mi situación de salud y eso ha tenido un impacto en mi familia, salud y académico».

En la dimensión de facilitador, las respuestas que brindan los estudiantes en relación a si el asesor de riesgo que le asignaron satisfizo todas sus inquietudes académicas, unánimemente es afirmativa, considerando 
que el asesor asignado en cada caso fue amable, confiable y empático, entre otras cualidades.

«Sí, eso sí, se tomó el tiempo para explicarme, fue empático, lo reconozco».

Al preguntar si los estudiantes se sintieron apoyados académicamente por el asesor para superar la situación de riesgo, generó dos tipos de respuestas, por un lado consideran que sí sintieron el apoyo y, sin embargo, por otro algunos estudiantes señalan que el asesor solo se enfoca en la obtención de una nota aprobatoria por parte del estudiante.

Respecto al perfil que debe tener el asesor de riesgo, la mayoría señala que debe ser amigable, comprensivo y empático, que conozca acerca del reglamento de la universidad, que conozca a los estudiantes que asesora para que sepa cómo ayudarlos a salir de la condición de riesgo, más aún, que no solo se evidencie su dominio académico, sino que se refleje su calidad humana.

«Amigable, compañera, más que todo para ser asesor de riesgo debe saber lo del reglamento y todo eso y más que todo debe ser amigable y comprensible, que se le comprenda».

Finalmente, en cuanto a la dimensión de sugerencias, se encuentra que a los estudiantes les gustaría contar con más apoyo sobre estrategias que les ayuden a salir del riesgo, así como contar con más cantidad de horarios disponibles de tal modo que no haya interferencias con las clases.

Otro aspecto importante que sugieren los estudiantes es que las asesorías, frente a la condición de riesgo académico, no se centren solamente en que el estudiante alcance una nota determinada, sino que enfaticen, por un lado, en aspectos más académicos como, por ejemplo, cómo comprender y asimilar las clases, si el profesor se muestra disponible ante las preguntas que se le formula; $y$, por otro lado, en aspectos más humanos, por ejemplo, por qué circunstancias está atravesando el estudiante, de tal manera que el abordaje sea más completo e integral.

\section{Estudiantes que no asisten al programa de asesoría de riesgo académico y superan dicha condición}

En referencia a la dimensión general, respecto a las razones por las cuales los estudiantes creen que han estado en la condición de riesgo académico, se encontraron diversasrespuestas, tales como: por problemas familiares, falta de organización del tiempo, falta de dedicación a los estudios y por la exigencia académica de los exámenes.

«...estoy en riesgo porque tengo problemas, bueno un poco de todo, es que soy madre soltera y es un poco difícil para mí».

Con respecto a la percepción sobre las facilidades que les brinda la universidad para poder superar la condición de riesgo académico, la mayoría de los estudiantes respondió de modo afirmativo, ya que recibieron mensajes a través de sus correos personales donde les señalaban que podían contar con un asesor, además de proporcionarles materiales y ambientes disponibles para poder recibir las asesorías y superar la condición de riesgo.

«Sí, por supuesto que sí porque tengo todo lo que necesito como ambientes, asesores y todos los materiales como para poder superar sin ningún problema».

Solo un estudiante señaló que no sabía con certeza si la universidad brindaba facilidades para superar la condición de riesgo académico. 
"La verdad no sé, porque yo he pasado el curso porque a mí me han dado las ganas de pasarlo y me he puesto las pilas, y me he recuperado de un bajón que tuve familiar, o sea nunca he recurrido a una ayuda de la universidad para pasar un curso».

En relación a las razones por las cuales los estudiantes decidieron no asistir al servicio de asesoría de riesgo académico, se halló que la mayoría señala que ha sido por falta de tiempo, interferencias entre los horarios asignados en las asesorías y los trabajos, e interferencias por roles que cumple el estudiante como, por ejemplo, ser padre/madre de familia.

\section{«...no pude asistir por falta de tiempo».}

Solo un estudiante señaló que no asistió porque pensó que podía superar la condición de riesgo solo.

«...no participé porque creí que lo podía manejar por mi cuenta».

Con respecto a las estrategias que emplearon los estudiantes para superar el riesgo académico, refirieron: dedicarse más a los estudios esforzándose de manera sostenida, tener confianza al profesor, ser más responsable, organizar mejor su tiempo y asistir a algunos talleres que puedan ser útiles.

\section{Estudiantes que no asisten al programa de asesoría de riesgo académico y no superan dicha condición}

Acerca de la dimensión general, ante la pregunta formulada a los estudiantes respecto a las razones por las cuales consideran que estuvieron en riesgo académico, la mayoría señala que ha sido por falta de organización, por problemas personales, por falta de dedicación a los estudios, porque algunos cursos son demasiado difíciles y por la interferencia producida por las amistades.
«... Yo creo que estoy en riesgo por mi falta de organización».

«... Estuve en riesgo porque la verdad es que tuve muchos problemas personales que no permitieron el ciclo pasado tomarme en serio la universidad y entonces no estudié mucho, bajé mucho mis promedios...».

«... El motivo por el que estoy en riesgo es por falta de organización, porque me distraje mucho con mis amigos».

Por otro lado, en relación al apoyo brindado a los estudiantes por parte de la universidad, consideran que esta les brinda las facilidades necesarias para poder superar la condición de riesgo académico, indicando que la institución educativa brinda todas las herramientas para poder estudiar en forma adecuada, valoran el sistema de asesorías que existe, y además reconocen que ofrece diversas opciones para aquellos que están en riesgo.

Bueno, sí, yo considero que sí, porque ayudan al alumno que está en riesgo... 0 sea, para que no le den de baja hay opciones también como para que se pueda retirar y así se pueda evitar que lo saquen.

Entre los motivos que los estudiantes señalan como decisivos para no asistir a las asesorías de riesgo, se encuentra la falta de tiempo. Otra de las razones que aluden es por la influencia negativa de personas, exceso de confianza, pensaron que podían superar dicha condición solos y no lo lograron.

«No asistí porque me dejé llevar por muchas personas, me confié en mí mismo y pensé que podía organizar bien mis cosas y nо pude».

En relación a las estrategias que utilizaron los estudiantes para superar de alguna manera el riesgo académico, se hallaron diversas respuestas, algunos indican que han 
intentado dedicar más tiempo a los estudios para poder alcanzar notas satisfactorias y subir el ponderado. Otros señalan que la estrategia es retirarse del curso.

«Sí lo intenté, primero evalué el número de cursos y la dificultad, y me propuse dedicarle más tiempo a mis estudios».

Referente a la pregunta de cuál creen los estudiantes que fue el motivo principal por el que no lograron superar la condición de riesgo académico, se encontró que identifican diversas razones, como la falta de estrategias, la carencia de una verdadera vocación por la carrera elegida, la desorganización, la ausencia de información sobre retiros de cursos que se pueden hacer antes de los exámenes parciales, el incumplimiento de la asistencia y puntualidad (faltas y tardanzas), la interferencia producida por las amistades y el exceso de confianza.

En cuanto a la dimensión de sugerencias, se encontró que algunos estudiantes señalaron que las asesorías deberían incrementarse en algunas sedes y cambiarse el sistema, de tal modo que cualquier asesor ayude a cualquier estudiante y no sea por especialidad.

\section{DISCUSIÓN}

Este estudio responde a las necesidades establecidas en la literatura sobre la necesidad de la evaluación y mejora continua de los programas de corte tutorial con el fin de que respondan a las necesidades que presentan los estudiantes en el contexto de la universidad en que estudian, de tal forma que se garantice su efectividad (Ariza \& Ocampo, 2005). Asimismo, se indaga sobre los aspectos sugeridos acerca de temas importantes de evaluar en los programas tutoriales como: los factores valorados en el tutor, las necesidades que debe cubrir y las propuestas de mejora (Gil-Albarova et al., 2013).
Con respecto a lo encontrado, en líneas generales se puede observar que los alumnos, asistentes y no asistentes al servicio de asesoría de riesgo académico tienen una percepción positiva del servicio, sobre todo en lo referido al apoyo que les brinda la institución para poder salir de la condición de riesgo y en la disposición de ayuda y buen trato de los asesores de riesgo. Estos puntos son importantes de señalar, ya que pueden contribuir a que la experiencia en la universidad de estos alumnos en riesgo académico sea positiva (Hojaset al., 2012), a pesar de las dificultades académicas que puedan presentar en un momento determinado de la carrera o si deciden posponer sus estudios.

Con respecto a las razones que los estudiantes atribuyen a su condición de alumnos en riesgo, casi todos coinciden en una organización inadecuada del tiempo, así como los problemas personales y familiares. Las razones señaladas por los estudiantes coinciden con lo encontrado en referencia a los factores que afectan el rendimiento académico de los universitarios (Mercado \& Niño, 2012; Artunduaga, 2008; Montero et al., 2007; Tejedor \& García-Valcárcel, 2005). En este sentido, es importante señalar que también la mayoría de alumnos coincide en que justamente estos son los temas que se trabajan en las asesorías de riesgo académico. Además, existe una mayor coincidencia en este punto con los alumnos que asistieron al servicio y superaron el riesgo, con lo que se podría afirmar que los asesores de este grupo en particular brindan estrategias que ayudan a los alumnos a mejorar su rendimiento académico porque toman en cuenta las necesidades de los mismos.

No obstante, también existen testimonios de alumnos que piensan que no se les han brindado las estrategias que ellos necesitaban. Es de vital importancia compartir este punto con los asesores de riesgo para que sus 
intervenciones apunten siempre a la necesidad particular que trae el alumno, de esta forma la ayuda brindada en el servicio podría ser más efectiva.

Otra de las intervenciones que los alumnos resaltan como efectivas, no solo en el proceso de asesoría sino que trasciende más allá de la misma, es que el asesor de riesgo los haya estimulado para la mejora de su rendimiento académico. Esto coincide con lo encontrado por Mercado y Niño (2012), quienes señalan que en los estudiantes con bajo rendimiento académico hay menor presencia de motivación de tipo intrínseca.

Otro punto en que los estudiantes hacen hincapié es en la importancia de la orientación que se les debe brindar sobre los procesos administrativos indicados en el reglamento de estudios de la universidad a los que ellos pueden acceder como alumnos en riesgo (retiro de curso, retiro de ciclo, etcétera). A pesar de que algunos afirman haber recibido esta orientación por parte de los asesores, otros sugieren que esta orientación pueda ser más completa $\mathrm{y}$ profunda. Esta necesidad planteada por los alumnos es válida y necesaria de implementar, ya que, como señalan Ariza y Ocampo (2005), los programas tutoriales deben contemplar e incluir las políticas y normativas en las que se enmarca la universidad.

Por otro lado, casi todos los alumnos entrevistados coinciden en la preferencia de las asesorías personalizadas e individuales por permitir un contacto más directo entre el alumno y el asesor, lo que a su vez facilita la comprensión entre ambos. En esta misma línea, Argüis et al. (2001 en Ariza \& Ocampo, 2005) indican que las tutorías individuales facilitan el conocimiento del alumno por parte del tutor.

Por otro lado, si bien es cierto los alumnos reconocen que las asesorías les brindan ayuda para superar el riesgo académico, también señalan que depende de ellos mismos el salir o no de dicha condición. Esta afirmación, aunque parezca contradictoria, se relaciona con lo expresado por el grupo de alumnos que indica como un punto negativo que en las asesorías solo se les brinda información y deben ser ellos los que tomen la decisión final. Esta relación se establece en el sentido de que en ambos casos se estaría promoviendo la toma de decisiones independiente y la autonomía en los alumnos, que son dos puntos claves considerados en los objetivos y lineamientos generales del servicio de asesoría de riesgo académico. Lo anterior coincide con lo sugerido por Tejedor y GarcíaValcárcel (2005), quienes indican que en las tutorías se deben promover las actitudes de responsabilidad, esfuerzo y autoexigencia en los alumnos.

Los estudiantes señalan algunas sugerencias para el servicio, como que se les brinde siempre estrategias específicas, apoyo psicológico, que indaguen sobre la forma cómo se está dirigiendo el curso, un seguimiento más cercano y continuo por parte del profesorasesor, entre otros. Estos puntos en particular, señalados por los alumnos, son de vital importancia para conocer sus requerimientos y necesidades y poder responder a los mismos.

El pedido de los alumnos que se les brinden estrategias específicas en las asesorías de riesgo es válido y posible, ya que, si bien es cierto, los estudiantes que atiende este servicio provienen de diversas carreras y diversos ciclos académicos, es factible potenciar en ellos estrategias académicas generales de ayuda para todo estudiante universitario, que colaboren con su proceso de aprendizaje. Marugán, Martín, Catalina y Román (2012) afirman que este tipo de estrategias ayudan al estudiante en el incremento de la autorregulación en el aprendizaje y mejoran su toma de conciencia y 
el control sobre lo que se va aprender, la calidad del aprendizaje y, por ende, el rendimiento académico. Es muy posible, además, que estos alumnos tengan este pedido en específico, ya que se ha encontrado que los alumnos con bajo rendimiento académico manejan estrategias que los conducen a un aprendizaje de menor profundidad en comparación con los alumnos que mantienen un buen rendimiento académico (Gargallo, Suárez, García, Pérez \& Sahuquillo, 2012).

Específicamente en lo que respecta al apoyo psicológico, es necesario en la medida en que en muchas ocasiones las dificultades a nivel académico pueden generar correlatos afectivos que ocasionen aún mayores dificultades en los alumnos para salir de la condición de riesgo académico; por ejemplo, se ha encontrado que, aunque el estado emocional del estudiante no es determinante en su rendimiento académico (Trógolo \& Medrano, 2012), la ansiedad que pueda experimentar juega un papel muy importante en el momento en que realiza un examen, ocasionando sufrimiento en él. Además, se han encontrado niveles más elevados de ansiedad en los estudiantes que dedican menos tiempo a prepararse para un examen en comparación con los que dedican más tiempo (Álvarez, Aguilar \& Lorenzo, 2012), grupo en el que probablemente estén inmersos los estudiantes en riesgo académico, ya que han reportado su falta de habilidad para organizar su tiempo adecuadamente.

En relación al seguimiento más cercano por parte de los profesores, sugerido por los alumnos, en primer lugar es importante señalar que los estudiantes que se apoyan con ayuda de profesores presentan mejor rendimiento académico que los que no lo hacen, siendo necesario el seguimiento de los estudiantes con resultados académicos deficientes, contando para ello con la asesoría permanente que deben brindar los docentes de la universidad; así como también ofrecerles apoyo en otras áreas como la social, personal, pedagógica (Mercado \& Niño, 2012). En este sentido, es necesario promover que todos los estudiantes que asistan al servicio se sientan de esta manera, ya que al responder a las necesidades planteadas por los alumnos se podría lograr que mayor cantidad de estudiantes hagan uso del servicio.

De lo anterior, señalado por los universitarios, también se desprende la importancia de indagar sobre los procesos de enseñanza utilizados por los docentes al desarrollar las actividades académicas en el aula y fuera de ella, no solo por ser un tema de interés para los alumnos, sino también porque se ha encontrado que estos son factores asociados al rendimiento académico y satisfacción de los estudiantes (Mercado \& Niño, 2012; Küster \& Vila, 2012).

Finalmente, en relación a los alumnos que no asisten a las asesorías, se encuentra por un lado que los que superaron la condición de riesgo manejaron estrategias específicas que fueron eficaces en anular dicha condición, como el esfuerzo sostenido, promover mayor confianza en su relación con el profesor, ser más responsable, organizar mejor su tiempo y asistir a algunos talleres de apoyo; en cambio, los alumnos que no asistieron y no superaron la condición de riesgo no manejan estrategias específicas puesto que les es difícil identificarlas y nombrarlas, además de ser el único grupo en señalarse como «confiados» o pensar que no necesitaban ayuda. Se puede observar, nuevamente, que el conocimiento y manejo de pautas y estrategias específicas, así como el nivel de autorregulación alcanzado (Garello \& Rinaudo, 2013), colaboran en que los estudiantes no solo superen la condición de riesgo sino mejoren su rendimiento académico. Es importante, por ello, promover la enseñanza y práctica de estrategias específicas, 
según cada caso particular, en los alumnos que utilizan el servicio.

\section{CONCLUSIONES}

En base a los hallazgos encontrados se hace evidente la necesidad de la evaluación continua no solo de los servicios de corte tutorial, sino de todos los programas que estén destinados a la orientación de estudiantes, ya que es la única forma de plantear y llevar a cabo mejoras que respondan a sus necesidades, situación que se confirma en los resultados obtenidos.

Si bien es cierto, estos resultados reflejan exclusivamente las percepciones de los participantes de una institución de educación superior en particular; es importante considerar que se han encontrado características que los alumnos en riesgo académico comparten con los estudiantes con bajo rendimiento académico en diversos estudios señalados previamente. Es por ello que, aunque los resultados no se puedan trasladar de forma directa a otras realidades, sí se crea un precedente en cuanto a que los universitarios tienen la necesidad de expresar su opinión y necesidades con respecto a los servicios que reciben en la casa de estudios.

Para el caso específico de los alumnos en riesgo académico de la Universidad Peruana de Ciencias Aplicadas, existe una percepción general positiva del servicio de asesoría y señalan componentes que son necesarios incorporar a la asistencia que se les brinda. En esta línea, el enfoque cualitativo aporta de forma especial, ya que permite conocer de manera profunda y particular las experiencias y percepciones de los usuarios.

Asimismo, es importante prestar especial atención a los aspectos positivos resaltados por los alumnos con el objetivo de mantenerlos y reforzarlos, ya que son ellos los que facilitan el acercamiento al servicio y la satisfacción actual con el mismo. En este sentido, al ser los profesores asesores de riesgo, uno de los elementos positivos señalados es la importancia de mantener la calidad de la atención a través de acciones como una selección cuidadosa y exhaustiva de las mismas, y procurar su capacitación permanente.

Por otro lado, sería interesante que en próximos estudios se incluya la participación de los asesores de riesgo, ya que al ser ellos quienes escuchan de primera mano las necesidades de los alumnos y quienes deben responder a ellas pueden brindar información relevante para mejorar el servicio, así como reportar también sus necesidades en su labor de tutores (Lobato, Del Castillo \& Arbizu, 2005).

\section{REFERENCIAS}

Álvarez, J., Aguilar, J. \& Lorenzo, J. (2012). La ansiedad ante los exámenes en estudiantes universitarios: relaciones con variables personales y académicas. Electronic Journal of Research in Educational Psychology, 10(1), 333-354. Recuperado de http://repositorio.ual. es/jspui/bitstream/10835/1681/1/ Art_26_702.pdf

Arbizu, F., Lobato, C. \& Del Castillo, L. (2005). Algunos modelos de abordaje de la tutoría universitaria. Revista de psicodidáctica, 10(1), 7-21.

Ariza, G., \& Ocampo, H. (2005). El acompañamiento tutorial como estrategia de la formación personal y profesional: Un estudio basado en la experiencia de educación superior. Universitas Psychologica, 4(1), 31-40.

Artunduaga, M. (2008). Variables que influyen en el rendimiento académico en la universidad. Madrid: Departamento de Métodos de Investigación y Diagnóstico 
en Educación de la Universidad Complutense de Madrid. Recuperado de http://www.ori.soa.efn.uncor. edu/?publicaciones = variables - que influyen-en-el-rendimiento-academicoen-la-universidad

Cardona, B., Ramírez, M., \& Tamayo, M. (2011). El estudiante recién llegado en el escenario universitario. Revista Lasallista de Investigación, 8(2), 76 - 88.

Creswell, J., Hanson, W., Clark, V. \& Morales, A. (2007). Qualitative research designs: Selection and implementation. The Counseling Psychologist, 35(2), 236-264.

Fernández, G., \& Escribano, M. (2008). Las tutorías en la formación académica y humana de los alumnos en la Universidad San Pablo CEU. Madrid: Departamento de Métodos Cuantitativos de la Universidad de San Pablo. Recuperado de http://www.uv.es/asepuma/ XVI/605.pdf

Garello, M. \& Rinaudo, M. (2013). Autorregulación del aprendizaje, feedback y transferencia de conocimiento. Investigación de diseño con estudiantes universitarios. Revista electrónica de investigación educativa, 15(2), 131-147. Recuperado de http:// redie.uabc.mx/index.php/redie/ article/view/451

Gargallo, B., Suárez, J., García, E., Pérez, C. \& Sahuquillo, P. (2012). Enfoques de aprendizaje en estudiantes universitarios excelentes y en estudiantes medios. Revista española de pedagogía, 70(252), 185-200.

Gil-Albarova, A., Martínez, A., Tunnicliffe, A. \& Moneo, J. (2013). Estudiantes universitarios y calidad del Plan de acción tutorial. Valoraciones y mejoras. Revista de docencia universitaria, 11(2),
63-87.

Herrera-Torres, L. \& Lorenzo-Quiles, 0. (2009). Estrategias de aprendizaje en estudiantes universitarios. Pedagogía universitaria, 12(3), 75-98.

Himmel, E. (2002). Modelos de análisis de la deserción estudiantil en la educación superior. Calidad en la educación, 17(2), 91-108.

Hojas, A., Anais, M., Bustos, A., Letelier, C. \& Zuzulich, S. (2012). Requerimientos académicos en estudiantes universitarios: el camino recorrido por el centro de apoyo al rendimiento académico y de exploración vocacional de la UC. Calidad en la Educación, 36, 249-263.

Küster, I. \& Vila, N. (2012). El docente universitario y sus efectos en el estudiante. Estudios sobre educación, 23, 157-182.

Lobato, C., Del Castillo, L. \& Arbizu, F. (2005). Las representaciones de la tutoría universitaria en profesores y estudiantes: estudio de un caso. International Journal of Psychology and Psychological Therapy, 5(2), 148-168.

Marugán, M., Martín, J., Catalina, J. \& Román, J. (2012). Estrategias cognitivas de elaboración y naturaleza de los contenidos en estudiantes universitarios. Psicología educativa, 19, 13-20.

Malbrán, M. (2004). La tutoría en el nivel universitario. Revista de Informática Educativa y Medios Audiovisuales, 1(1), 5-11.

Mercado, T.\&Niño,C.(2012). Factoresacadémicos $\mathrm{y}$ personales asociados al rendimiento académico de los estudiantes del Programa de Administración de Empresas de la Universidad de Sucre. Zona Próxima, 16, 54-67. 
Montero, E., Villalobos, J. \& Valverde, A. (2007). Factores institucionales, pedagógicos, psicosociales y sociodemográficos asociados al rendimiento académico en la Universidad de Costa Rica: Un análisis multinivel. Relieve, 13(2), 215-234.

Mori, M. (2012). Deserción universitaria en estudiantes de una universidad privada de Iquitos. Revista Digital de investigación en Docencia Universitaria, 6(1), 60-83. Recuperado de http://www3.upc.edu. pe/docencia-universitaria/Det_Bol1.asp ?CON=9058\&BOL=16\&EJE $=789 \&$ SEC $=\mathrm{A}$ rt\%EDculos

Muñoz, F. (1999). El estrés académico: incidencia del sistema de enseñanza y función moduladora de las variables psicosociales en la salud, el bienestar y el rendimiento de los estudiantes universitarios. (Tesis doctoral, Universidad de Sevilla). Recuperado de http://fondosdigitales. us.es/tesis/tesis / 669/el-estresacademico-incidencia-del-sistema-deensenanza-y-funcion-moduladora-delas-variables-psicosociales-en-la-saludel-bienestar-y-el-rendimiento-de-losestudiantes-universitarios/

Tejedor, F. \& García-Valcárcel, A. (2005). Causas del bajo rendimiento del estudiante universitario (en opinión de los profesores y alumnos). Propuestas de mejora en el marco del EEES. Revista de Educación, 342, 443-473.

Trógolo, M. \& Medrano, L. (2012). Personality traits, difficulties in emotional regulation and academic satisfaction in a sample of argentine college students. International Journal of Psychological Research, 5(2), 30-39.

Universidad Peruana de Ciencias Aplicadas (2013a). Reglamento de estudios (SICAREG-05). Recuperado del sitio de internet de la Universidad Peruana de
Ciencias Aplicadas, Centro de Atención al Alumno: http://sica.upc.edu.pe/ sites/sica.upc.edu.pe/files/SICA-REG05-REGLAMENTO-DE-ESTUDIOS.pdf

Universidad Peruana de Ciencias Aplicadas (2013b). Manual del Tutor (OA-P-01). Recuperado del sitio de internet de la Universidad Peruana de Ciencias Aplicadas, Área de Calidad Educativa: http://sica.upc.edu.pe/sites/sica. upc.edu.pe/files / OA-P-01\%20 MANUAL\%2 0DEL\%2 0 TUTOR\%20 04062013.pdf 